numbers are punched retrospective from 2010. Information on alcohol and drugs was found in the free text variable. Definition of Recidivism: In some studies more than two injury episodes. The present study includes five or more. Aim of study is identification of target groups and tailoring community interventions.

Results Out of 27090 records on 18667 persons, we identified 360 treated for five or more injury episodes. These 360 were treated in 2321 different injury treatment episodes. The range of episodes was five to 41 . Overrepresented groups were: 1) females age 19-29, treated for self-harm, alcohol/drugs/narcotics involved in most cases 2) males age group 19-29, treated for interpersonal violence, also with stories including alcohol and drugs 3) elderlies falling, 4) adolescent and young men in high energy activities.

Conclusions A continuous hospital based injury registry can identify characteristics and distribution of injury recidivists in a population.

\section{RECENT TRENDS OF ROAD TRAFFIC FATALITIES IN CHINA: COMPARING NATIONAL AND SUBNATIONAL DATA SOURCES}

Qingfeng Li, Huan He, Hailun Liang, David M Bishai, Adnan A Hyder. Johns Hopkins International Injury Research Unit, Department of International Health, Johns Hopkins Bloomberg School of Public Health

\subsection{6/injuryprev-2016-042156.315}

Background This study aims to better understand public data sources and evaluate recent trends in road traffic fatalities (RTF) in China.

Methods We systematically reviewed and compared multiple national sources of RTF data. These included: population-based reports from the Ministry of Public Security (MPS), samplebased estimates from the vital registration system of Ministry of Health (MOH) and the Disease Surveillance Points System (DSP), as well as model-based estimates from the World Health Organisation (WHO) and the Global Burden of Disease Study (GBD). A comprehensive review of publications focused on subnational RTF was also conducted. We based our assessment of the recent trend of RTF on using fixed effects and random effects longitudinal models.

Results Despite the discrepancies in estimates, all national data sources indicated that RTF had been increasing prior to 2005. Since then, the MPS reports indicate a declining trend, DSP estimates showed a flat trajectory while estimates from $\mathrm{MOH}, \mathrm{GBD}$ and WHO continue to show increases in RTF. Data from local death registration systems are largely consistent with MPS reports.

Conclusions Reflecting a non-representative sample, $\mathrm{MOH}$ data were used in generating model-based estimates by the WHO and GBD; consequently, the trends from these sources are open to.

MPS and local independent jurisdictional health data indicate that RTF have been declining since 2005. This may be consistent with recent interventions to reduce RTF. China's experience may be of great value for other developing countries though RTF still impose a tremendous health burden in the country.

\section{6 THE ROLE OF SELEKTION BIAS IN A CASE-CROSSOVER STUDY ON OCCUPATIONAL INJURIES}

${ }^{1}$ Anna H Oesterlund, ${ }^{2}$ Flemming Lander, ${ }^{3}$ Søren Rytter and ${ }^{1}$ Jens M Lauritsen. ${ }^{1}$ Accident Analysis Group, Orthopaedic Department, Odense University Hospital and Institute of Clinical Medicine, University of Southern Denmark, Denmark; ${ }^{2}$ Department of Occupational and Environmental Medicine, Odense University Hospital, Denmark; ${ }^{3}$ Department of Orthopaedics, University Clinic for Hand, Hip, and Knee Surgery, Regional Hospital West Jutland, Denmark

\subsection{6/injuryprev-2016-042156.316}

Background The case-crossover studies in injury epidemiology research are gaining ground. The method compares transient exposure during intervals when an outcome occurs, to exposures during intervals without outcome for the same individual. Although non-responding is a concern in all interviewed based studies, previous studies have not involved and discussed the importance of selection as a source of bias that could influence the overall reliability of the risk outcomes. Due to the unique Danish identification number it is possible to examine data between responders and non-responders in our case-crossover study concerning injuries, industry, sex and age.

Methods The population base for the study was derived from two public Hospital Emergency Departments in Denmark. All contacts due to occupational injuries during 2013 were included. An occupational injury was based on asking the patient whether the injury occurred during paid work. All such injured patients were asked to participate. Those confirming participation (responders) were compared with those not responding or denying (non-responders).

Results Among all 4002 injured, 1693 were responders (42\%). The overall tendency of sex, age and distribution of injury were the same between both responders and non-responders except for "other types of injuries". When dividing into minor and major injuries there were no difference between responders and non-responders. Further investigation of potential bias for subgroups or industries awaits further analysis.

Conclusions So far, our results indicate no serious selection bias in sex, age and injury distribution in spite of low participation rate, and thus, provide good possibility for broader generalisation of the risk outcomes. This indicates a strengthening of the overall reliability of the risk outcomes from our and previous case-crossover studies.

\section{NON-FATAL INJURIES UNTREATED AT HOSPITALS IN HUNAN, CHINA: IMPLICATIONS FOR HOSPITAL-BASED SURVEILLANCE SYSTEMS}

${ }^{1}$ Yue Wu, ${ }^{2}$ Wei Zhang, ${ }^{2}$ Lin Zhang, ${ }^{3}$ David Schwebel, ${ }^{2}$ Peishan Ning, ${ }^{2}$ Xunjie Cheng, ${ }^{4}$ Xin Deng, ${ }^{2} \mathrm{Li} \mathrm{Li},{ }^{2}$ Jing Deng, ${ }^{1 \#}$ Guoging Hu. ${ }^{1}$ Department of Occupational and Environmental Health, School of Public Health, Central South University; ${ }^{2}$ Department of Epidemiology and Health Statistics, School of Public Health, Central South University; ${ }^{3}$ Department of Psychology, University of Alabama at Birmingham; ${ }^{4}$ Xiangya Hospital, Central South University

\subsection{6/injuryprev-2016-042156.317}

Background Disability adjusted life year (DALY) is among the most commonly-used indicators of disease/injury burden. It relies on accurate data concerning non-fatal conditions, which may be collected through hospital-based surveillance or population-based 
surveys. To determine whether hospital-based surveillance captures all non-fatal injuries, we assessed the extent of non-fatal but serious injuries not treated at hospitals.

Methods Data from the first provincial health household interview survey of Hunan, China, conducted in 2013, were used. Injury events were identified when any of the following circumstances occurred in the prior 14 days: (1) hospital visit following an injury; (2) receiving medical treatment elsewhere for an injury (e.g., taking medications, or receiving massage or hot compress); and/or (3) being off work or school, or in bed for more than 1 day, following an injury. We calculated the proportion of injury events not treated at hospitals and reasons for not visiting hospital for injury events occurring during the previous two weeks.

Results We captured 108 injury events (56 during the previous two weeks and 52 at other times). The weighted injury prevalence was 4.9 per 1,000 persons during the last two weeks $(95 \%$ confidence interval: $2.9-6.9$ per 1,000 persons). Of the 56 events, 14 (weighted proportion $41.2 \%$ ) were not treated at hospitals. Primary explanations for skipping hospital visits included perceiving injuries were too minor and economic limitations to travel to hospitals or seek treatment.

Conclusions Results imply the burden of non-fatal injury may be underestimated by hospital-based surveillance systems such as that used in China.

\section{Thematic Conference: "From Occupational Safety and Health Strategies to Practice"}

\section{Underreporting of Occupational Accidents (Reports on the NDPHS/EG OSH RealOcc Accidents Project)}

\section{\begin{tabular}{|l|l}
\hline 318 & RELIABILITY OF WORK INJURY STATISTICS IN THE BSN
\end{tabular} COUNTRIES - DECISION MAKING BASED ON PARTIAL FACTS?}

Kari Kurppa. Finnish Institute of Occupational Health, Finland

\subsection{6/injuryprev-2016-042156.318}

Indicators of work injuries, arising from national monitoring systems, are important outcome measures of occupational health and safety. However, underreporting of non-fatal work injuries (>3 days absence from work) is known to be common. Eurostat has pointed out to large differences between countries in the reporting level.

According to Eurostat, the reporting is close to $100 \%$ in the countries which have a work injury insurance system (Finland, Germany) in which the compensation of work injuries is more generous than that of non-occupational injuries. In Denmark, Norway, Sweden which have a universial social security coverage and similar compensation for occupational and non-occupational injuries, the reporting level has been much lower than expected.

In the formerly socialist BSN countries (Estonia, Latvia, Lithuania, Poland, Russian Federation), the level of reporting of work injuries is mostly unknown. Yet, high rates of fatal work injuries in these countries in combination with low rates of non-fatal injuries suggest that much of the non-fatal injuries remain unregistered.
Within the context of the Baltic Sea Network of Occupational Health and Safety (BSN) (Denmark, Estonia, Finland, Germany, Latvia, Lithuania, Russian Federation, Norway, Poland, Sweden) semi-quantitative analyses of the level of reporting work injuries were recently made. The results suggested that less than $10-20 \%$ of non-fatal work injuries were registered in many formerly socialist BSN countries.

Decision makers tend to trust that official statistics provide a reasonably correct appraisal of the state of affairs. It must be difficult to accept as true that, due to underreporting, the majority of work injuries, $80 \%$ or more in some countries, may not be included in the national statistics.

If only a small proportion of work injuries is registered in a country it is impossible to say with any degree of certainty what the actual situation is or is the situation developing for better or for worse.

\section{Parallel Sessions Tuesday 20.9.2016 16:30-18:00}

\section{Safe Communities, ESCON}

\section{Parallel Tue 3.1}

\section{A NEW NGO IN THE PAN PACIFIC REGION TO PROMOTE INJURY PREVENTION AND THE SAFE COMMUNITY MOVEMENT}

${ }^{1}$ Tania Peters, ${ }^{2}$ Deborah Costello, ${ }^{3}$ Carrie Nie, ${ }^{4}$ Pamela Fuselli. ${ }^{1}$ Safe Communities Foundation New Zealand; ${ }^{2}$ Australian Safe Communities Foundation; ${ }^{3}$ Safe Communities America; ${ }^{4}$ Parachute, Canada

\subsection{6/injuryprev-2016-042156.319}

Background The Pan Pacific Safe Communities Network represents almost 100 accredited Safe Communities - 1.5 million people in Australia, Canada, New Zealand and the United States. As an advocate for injury/violence prevention the PPSCN promotes evidence based programming, leadership, sustainability, evaluation training, mentors communities, conducts accreditations and provides networking opportunities

Description Injuries and violence are leading public health problems. Safe Communities promotes a structured and collaborative approach to their prevention. PPSCN was established due to changes in the centralised infrastructure of International Safe Communities. In 2014, PPSCN began pursuing official relations with the WHO to establish opportunities to work on specific projects to advance our mutual goals of developing sustainable, equitable and transferable violence and injury prevention models at the local level.

Results PPSCN has operated since 2010 (registered NGO 2013) is strongly committed to being a primary resource for Safe Communities by providing timely and effective mentoring, advice, advocacy, and accreditations. Each country in PPSCN is structured differently but dedicates resources to support the movement. PPSCN members are committed to best practice in violence and injury prevention through greater collaboration between non-government organisations, the business sector, and government agencies. Through the development of these collaborative relationships Safe Communities have been successful in growing and strengthen community safety activities to create safer environments and increase the adoption of safer behaviours. www.ppscn.org 